\title{
Das Arztgeheimnis bleibt!
}

\section{Monique Gauthey}

Fachärztin, Mitglied des Zentralvorstandes der FMH, Departementsverantwortliche Spitalärztinnen und -ärzte

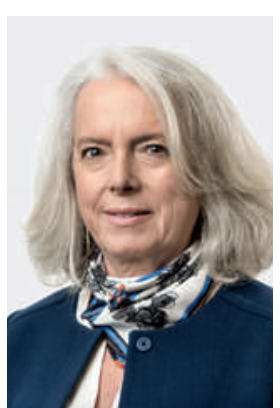

Obwohl die Gesetzeslage eindeutig ist, geraten Psychiaterinnen und Psychiater, die mit gefährlichen Strafgefangenen arbeiten, unter dem aktuellen politischen Druck ins Zweifeln. Dieser Druck, das Arztgeheimnis zu schwächen, lässt derweil nicht nach, auch wenn die Ärzte in ihrem Kampf breit unterstützt werden, insbesondere durch Anwälte und Richter. Seit Bestehen des Arztgeheimnisses wiederholt sich die Debatte, obwohl offenkundig noch keine bessere Lösung gefunden wurde, um allen Bevölkerungsgruppen $\mathrm{Zu}$ gang zu medizinischer Versorgung zu ermöglichen. Das Arztgeheimnis stellt im weitesten Sinn eine Massnahme zum Schutz der öffentlichen Gesundheit dar. Uns allen ist klar, dass unser Vertrauen zum behandelnden Arzt an dessen Verschwiegenheit gebunden ist; von diesem Vertrauen hängt die Bereitschaft ab, sich dem Arzt anzuvertrauen.

Natürlich könnten Ärztinnen und Ärzte auch ohne Arztgeheimnis weiter «behandeln», wobei allerdings der Zugang zu bestimmten Patienten oder bestimmten Beschwerden unmittelbar eingeschränkt wäre. Hierbei kann es sich um bestimmte "peinliche» Krankheiten handeln, um Patienten, die mit den Behörden im Kon-

\section{Wenn wir uns der Aushöhlung von Freiheit und Menschlichkeit nicht beharrlich widersetzen, riskieren wir weitere, noch schwerwiegendere Eingriffe.}

flikt stehen, wie etwa Regimegegner, aber auch um psychiatrische Pathologien. Diese Geheimnisse stellen für betroffene Patienten wichtige Geheimnisse dar, die es gegen alle Widerstände zu wahren gilt.

Dass die Psychiater meinen, in aller Transparenz mit Patienten arbeiten zu können, die von den Justizbehörden zur Behandlung gezwungen werden, reicht als Argument zur Änderung der rechtlichen Rahmenbedingungen nicht aus. Bei ergebnis- oder verhaltens- zentrierten Behandlungen ist ein solches Arbeiten sicherlich möglich, indem bestimmte Themen nicht angeschnitten werden. Die Situation ist aber völlig anders, wenn es darum geht, langfristig mit schwer devianten Verhaltensweisen und verborgenen Störungen zu arbeiten. Wie der Artikel von Prof. Sprumont und

\section{Sollte die Anwendung eines Gesetzes den Arzt} in einen Gewissenskonflikt bringen, muss der Arzt seinen Patienten verteidigen.

Prof. Ducor auf Seite 1592 zeigt, beschreibt das Gesetz zwei verschiedene Rollen - einerseits die des behandelnden Arztes und andererseits die des Experten. Durch die klare Trennung dieser beiden Rollen bleibt der therapeutische Rahmen gewahrt.

Die Diskussion berührt hier grundlegende Begriffe wie Freiheit und Gleichheit. Die Gleichwertigkeit der medizinischen Versorgung von Strafgefangenen ist ein unveräusserlicher Grundstein; in einem Rechtsstaat kann es keine Bürger geben, die weniger gleich sind als andere. Wenn wir uns der Aushöhlung von Freiheit und Menschlichkeit nicht beharrlich widersetzen, riskieren wir weitere, noch schwerwiegendere Eingriffe. Um es mit Benjamin Franklin auszudrücken: «Wer die Freiheit aufgibt, um Sicherheit zu gewinnen, wird am Ende beides verlieren.»

Dasselbe droht, wenn man die Ärztinnen und Ärzte dazu zwingt, das Gesetz über die Ethik zu stellen. Sollte die Anwendung eines Gesetzes den Arzt in einen Gewissenskonflikt bringen, muss der Arzt seinen Patienten verteidigen. Kehrte man diese Logik um, so gerieten die Ärzte in eine Sackgasse: Man könnte ihnen jederzeit und ohne Grundlage vorhalten, eine Tatsache nicht gemeldet zu haben, die nur ihnen bekannt ist. Vertrauen ist etwas sehr Zerbrechliches, und bereits eine einzige Verfehlung kann es zerstören. 